\title{
Infall variability in the Classical T Tauri system VZ Chamaeleonis
}

\author{
K. Smith ${ }^{1,2}$, G. F. Lewis ${ }^{3}$, I. A. Bonnell ${ }^{4}$, and J. P. Emerson ${ }^{5}$ \\ 1 Institut für Astronomie, ETH-Zentrum, 8092 Zürich, Switzerland \\ 2 Paul Scherrer Institut, Würenlingen und Villigen, 5232 Villigen PSI, Switzerland \\ 3 Anglo-Australian Observatory, PO Box 296, Epping, NSW 1710, Australia \\ e-mail: gfl@aaoepp.aao.GOV.AU \\ 4 University of St Andrews, St Andrews, Scotland \\ e-mail: iab1@st-andrews.ac.uk \\ ${ }^{5}$ Department of Physics, Queen Mary, University of London, Mile End Road, London E1 4NS, UK \\ e-mail: j.p.emerson@qmw.ac.uk
}

Received 11 June 2001 / Accepted 13 September 2001

\begin{abstract}
We present time series spectroscopy of the Classical T Tauri star VZ Cha. We follow spectral variations at intermediate resolution over five successive nights, or approximately two rotation periods. We see profile features which persist on timescales longer than the expected infall time from the inner disc, and we see expected evidence of rotational variations in the lines, but we also note that rotation alone cannot produce all the observed variability and some other mechanism must be invoked. The behaviour of $\mathrm{H} \alpha$ is observed to be markedly different from that of the other lines. In particular, the evidence of rotational effects is lacking at $\mathrm{H} \alpha$, and the activity in the red and blue wings of the line is not significantly correlated, in contrast to the other Balmer lines.
\end{abstract}

Key words. accretion - accretion disks - stars: activity, pre-main sequence, variables: general

\section{Introduction}

Classical T Tauri stars (CTTS) accrete material through circumstellar discs at a rate of $10^{-8}$ to $10^{-7} M_{\odot} \mathrm{yr}^{-1}$ (Gullbring et al. 1998). This accretion leads to strong infrared and ultraviolet excess continuum emission, and the formation of emission lines. Absorption features relative to the mean profile are often seen in the red wings of emission lines (Edwards et al. 1994) and indicate free-falling material in the line of sight (e.g. Bonnell et al. 1998). Coupled with various other factors such as generally rapid rotation rates, apparent star-disc rotation locking and the occasional presence of photospheric spots, these suggest that the inner part of the circumstellar disc is truncated by a strong stellar magnetic field and the accreting material is channelled down the field lines onto the star (Königl 1991). This picture is analogous to models developed for magnetic neutron star systems (e.g. Ghosh \& Lamb 1978 and following papers), and became more firmly established for CTTS with the detection of kiloGauss-strength magnetic fields in small samples of $\mathrm{T}$ Tauri systems (Basri et al. 1992; Guenther et al. 1999; Johns-Krull et al. 1999). The geometry and detailed nature of the magnetically funnelled accretion process remain poorly understood, and

Send offprint requests to: K. Smith, e-mail: kester@astro.phys.ethz.ch are of importance if we are to understand both accretion and related phenomena such as jets and outflows or the regulation of the star's angular momentum during pre-main sequence evolution. A crucial initial question is whether the field is a large-scale ordered dipole, presumably tilted with respect to the stellar rotation axis, or whether it has more complex structure, perhaps similar to scaled-up magnetic loops in the Solar corona. In the former case we expect to see either one or two distinct accretion streams pass through the line of sight in each rotation period. In the latter case, there could in principle be any number of loops. Solar active regions can persist for weeks and by analogy we would expect $\mathrm{T}$ Tauri magnetic structures to survive at least for timescales comparable to the rotation period.

Modelling the optical spectroscopic appearance of these regions, which will be ultimately necessary for comparison with spectral variations, is problematic as full radiative transfer models are needed. Line profile modelling has been undertaken in an attempt to reproduce the observed hydrogen line profiles (Hartmann et al. 1994; Muzerolle et al. 1998). Line profiles were modelled as being due to the velocity distribution of the infalling material in the magnetosphere. The profiles produced were in good agreement with observed profiles, although no wind component was considered and Stark broadening was needed 
to explain the very broad profile of $\mathrm{H} \alpha$. Temperatures in the magnetosphere would need to be of the order of $7000-10000 \mathrm{~K}$, and the heating mechanism required is not specified, although magnetic effects (i.e. reconnection) would be a reasonable assumption.

The line profiles, and in particular the redshifted absorption components, are typically found to be strongly variable, and this is usually explained as being caused by accretion streams crossing the observer's line of sight as the system rotates. This implies an azimuthallyasymmetric accretion geometry. The simplest such geometry would be a dipole inclined to the stellar rotation axis, a situation which might be expected in any case by analogy with the Sun. Together with knowledge of the rotation period provided by previous photometric work, these variations could in principle be used to reveal the detailed structure of the magnetically dominated inner regions. It seems that non-axisymmetric models are applicable in at least some cases. For example, Kenyon et al. (1994) observed photometric variability of the extremely active CTTS DR Tau which was modelled as being due to a hot spot on the surface. This spot was identified as the accretion shock at the base of an accretion stream, indicating either a tilted dipole or a non-global field geometry. Hessman \& Guenther (1997) observed quasi-periodic variations in the fluxes of the major emission lines of DR Tau and DG Tau, attributed also to rotational modulation of the emitting accretion shock. Smith et al. (1998) also observed DR Tau, and reported varying redshifted absorption, consistent with one of several previously detected photometric periods and therefore suggestive of a rotating accretion stream being carried through the observer's line of sight. Analysis of equivalent width variations from the same data set revealed time lags of several hours between the four lowest Balmer lines and $\mathrm{Ca}$ II H and $\mathrm{K}$, with the higher Balmer lines varying before the lower ones (Smith et al. 1999). In the rotating-streams scenario, this would indicate that the variations of the different Balmer lines are linked but spatially displaced in some way. The most likely explanation is that the proportion of flux in the Balmer lines varies along the accretion stream, with the higher lines having a larger proportion from close to the hot accretion shock on the stellar surface. There is some evidence for this from the line flux modelling of Muzerolle et al. (1998). The interpretation proposed was that the time lags were the signature of a stream moving over the limb, the hot dense regions near the star being seen first with decreasing occultation by overlying stream material, then disappearing over the limb.

Analysis of line profiles at high resolution for SU Aur was made by Johns \& Basri (1995). They reported evidence of both infalling and outflowing components, which seemed to vary periodically with a $180^{\circ}$ phase difference. The interpretation was an "egg-beater" model in which a rotating inclined stellar dipole field disrupts a disk and is responsible for powering a disk wind. This model resembles models proposed by Shu et al. (1994) and also Paatz \& Camenzind (1996) in which a dipole stellar field powers an outflow from the inner disc. A major outstanding question is how these disc wind models would behave if the dipole field were non-axisymmetric, in particular, whether the outflow would originate from only those regions of the inner disc edge where the stellar field was locally strongest.

MUSICOS observations of SU Aur allowed this object to be monitored at high spectral resolution continuously over 10 nights (Oliveira et al. 2000). Profile variability of the principal Balmer lines and also Na I and He I D3 (5876 $\AA$ ) was monitored. An approximate half period shift was found between the variation of the red and blue wings of $\mathrm{H} \beta$, indicating outflow and infall are approximately in antiphase. Smaller time lags were found between the He I line and the redshifted component of $\mathrm{H} \beta$, both supposedly formed close to the stellar surface. The authors attribute this to an eclipse of a twisted accretion stream. The twisting is then interpreted as being caused by the differential rotation between the star and inner disc creating toroidal field.

Despite these tentative successes in understanding $\mathrm{T}$ Tauri environments in terms of an inclined dipole scenario, the possibility remains that the spectral variations may be caused by non-rotational effects. Variable accretion rates or magnetic reconnection in the magnetosphere would be candidates to produce such variations. The buildup of toroidal field by shear between disc and star would need to be dissipated if the field geometry is to be maintained, which leads us to expect some reconnection, with associated plasma heating and hence variability. Meanwhile, a persistant problem in the channeled accretion scenario is the loading of material onto the field lines before it can fall onto the star. Simulations by Miller \& Stone (1997) illustrate this for an aligned dipole geometry. The field lines in the disc plane are forced to bow inwards by the pressure of accretion through the disc, creating a local potential minimum from which disc material cannot escape. Accretion through the disc proceeds to build up material in this potential well, until the accretion pressure overwhelms the field and the material accretes at the stellar equator. This process could perhaps be circumvented in a non-aligned dipole geometry, but the possibility of unsteady accretion, through this or another mechanism, remains. Examples of models based on variable accretion include a "beat frequency" model proposed by Smith et al. (1995), which is analogous to that envisaged for low mass $\mathrm{X}$-ray binaries, to produce quasi-periodically varying accretion from the inner disc. Ultchin et al. (1997) suggested a model in which blobs of disc material are dislodged from the disc by stellar magnetic field and orbit the star, impacting either on the star or disc and giving rise to variations.

The situation in the centre of $\mathrm{T}$ Tauri systems is therefore far from clear. The cartoon picture of the star having an ordered dipole field, which then channels smooth accretion flows onto the magnetic poles, is almost certainly a crude simplification, although it seems that elements of it are useful. The full picture is too complex for detailed modelling to be a viable strategy. We must therefore 
continue to glean clues to the morphology of the magnetosphere through further observations.

In the current study, we present spectroscopic observations of the CTTS VZ Cha over five consecutive nights, corresponding to two and a half times the rotation period. On some nights, we obtained several spectra and so we are able to comment on variations on timescales of hours. We observed extreme profile variability of several different types, including redshifted absorption and emission, blue shifted absorption and emission, and equivalent width variability. We describe this variability and attempt to decompose the profiles into distinct components. We then examine the behaviour of the line components in the context of variable accretion and rotational modulation pictures.

\section{VZ Cha}

VZ Cha is a Classical T Tauri star with a K6 type photosphere. It is located in the Chamaeleon low mass star forming region at a distance of approximately $160 \mathrm{pc}$. The apparent $V$ magnitude is 12.75 and highly variable (Herbig \& Bell 1988). The star is a strong $\mathrm{H} \alpha$ emitter. The continuum veiling has not been previously measured, but the lack of any absorption lines in our blue spectra suggests the star is heavily veiled. Intermediate-resolution spectroscopy by Appenzeller et al. (1983) revealed redshifted absorption at $\mathrm{H} \beta$. Higher resolution spectroscopy then established the presence of various interesting spectroscopic phenomena, such as He II emission (indicative of hot plasma), blue shifted emission at a velocity of $189 \mathrm{~km} \mathrm{~s}^{-1}$ at $\mathrm{H} \alpha$ and redshifted absorption in the Balmer lines at velocities of around $240 \mathrm{~km} \mathrm{~s}^{-1}$ (Krautter et al. 1990). Drissen et al. (1989) found linear polarization, indicating the presence of a circumstellar disc. A photometric period of 2.56 days was found by Batalha et al. (1998), which is at odds with previously reported photometric periods of around 7 days (Kappelman \& Mauder 1981; Mauder \& Sosna 1975) although it should be noted that these last two studies were not based on sufficient data to derive statistically significant periods, but rather found suggestive evidence of periods in lightcurves when examined by eye. A $K$-band speckle survey for binarity by Ghez et al. (1996) did not detect any close companion, the limiting flux ratio being approximately 1 for a sky separation down to $0.1 \operatorname{arcsec}(16 \mathrm{AU}$ at $160 \mathrm{pc})$.

\section{The observations}

The observations were carried out between $6 / 7$ and 10/11 May 1998 with the $2.3 \mathrm{~m}$ ANU telescope, at the Siding Spring observatory in New South Wales, Australia. The instrument used was the dual beam spectrograph. Gratings of 1200 lines/mm were employed in each arm. This allowed us to observe (at first order) the spectral region from approximately $3900 \AA$ to $4880 \AA$ in the blue, and from approximately $5680 \AA$ to $6640 \AA$ in the red arm. Because our sensitivity was greater in the red, we were able to obtain several exposures of the red spectrum for every blue exposure. The data were reduced using standard IRAF ${ }^{1}$ routines. The dispersion was approximately $0.5 \AA$ per pixel in each arm. The $F W H M$ of a typical unblended arc line was found to be approximately $2.0 \AA$. The wavelength scale was calibrated by reference to arc spectra observed during the run. The rms of the fits to the arc-lines was found to range between 0.08 and $0.15 \AA$.

No photometric standards were observed, since the observations were not carried out in photometric conditions. The continuum was therefore removed by fitting with a Chebyshev polynomial and dividing it out.

\section{Analysis}

Various types of profile variability were seen, including redshifted and blueshifted emission and redshifted absorption. This variability occured predominantly on night-tonight timescales. The clearest variation was the appearance of Inverse P Cygni (IPC) type profiles on nights three and five. The pixel by pixel standard deviation across the profiles of the six strongest lines was computed and is shown in Fig. 1. For comparison an average profile constructed by taking the mean of all the available spectra, excluding the minimum and maximum at each pixel to prevent unwanted effects due to outliers, is also shown. For the Balmer lines at least, the variability seems to be divided into two main components, one in the blue wing and one in the red wing. We estimate the red component to lie at a velocity relative to the line centre (measured from the average profile) of approximately $70 \mathrm{~km} \mathrm{~s}^{-1}$, a value which remains more or less constant from $\mathrm{H} \alpha$ through to $\mathrm{H} \delta$. The varying component in the blue wing steadily increases in velocity shift from around $50 \mathrm{~km} \mathrm{~s}^{-1}$ in the case of $\mathrm{H} \delta$ up to $130 \mathrm{~km} \mathrm{~s}^{-1}$ for $\mathrm{H} \alpha$. The shape of the average $\mathrm{H} \alpha$ profile appears to have a blue hump, indicating a wind component which is present to some extent most of the time. To further quantify this variability, we subtracted the average spectrum from each of the programme spectra to obtain a measure of the variable residual emission at different times.

\subsection{Description of line profile variations}

The spectra, average profiles, and residuals are plotted in Fig. 2. Variations on both sides of the profiles are seen. Below, we describe the features seen in the residual profiles. We note first that some care must be taken when describing lack of emission in a profile relative to the average profile. Where the actual profile dips below the continuum level (which in Fig. 2 is at the zero flux level), we can be sure that absorption is occuring. However, where there is a lack of flux relative to the continuum, but the spectrum does not dip below the continuum, we may be

\footnotetext{
${ }^{1}$ IRAF is distributed by the National Optical Astronomy Observatories, which are operated by AURA under cooperative agreement with the National Science Foundation.
} 


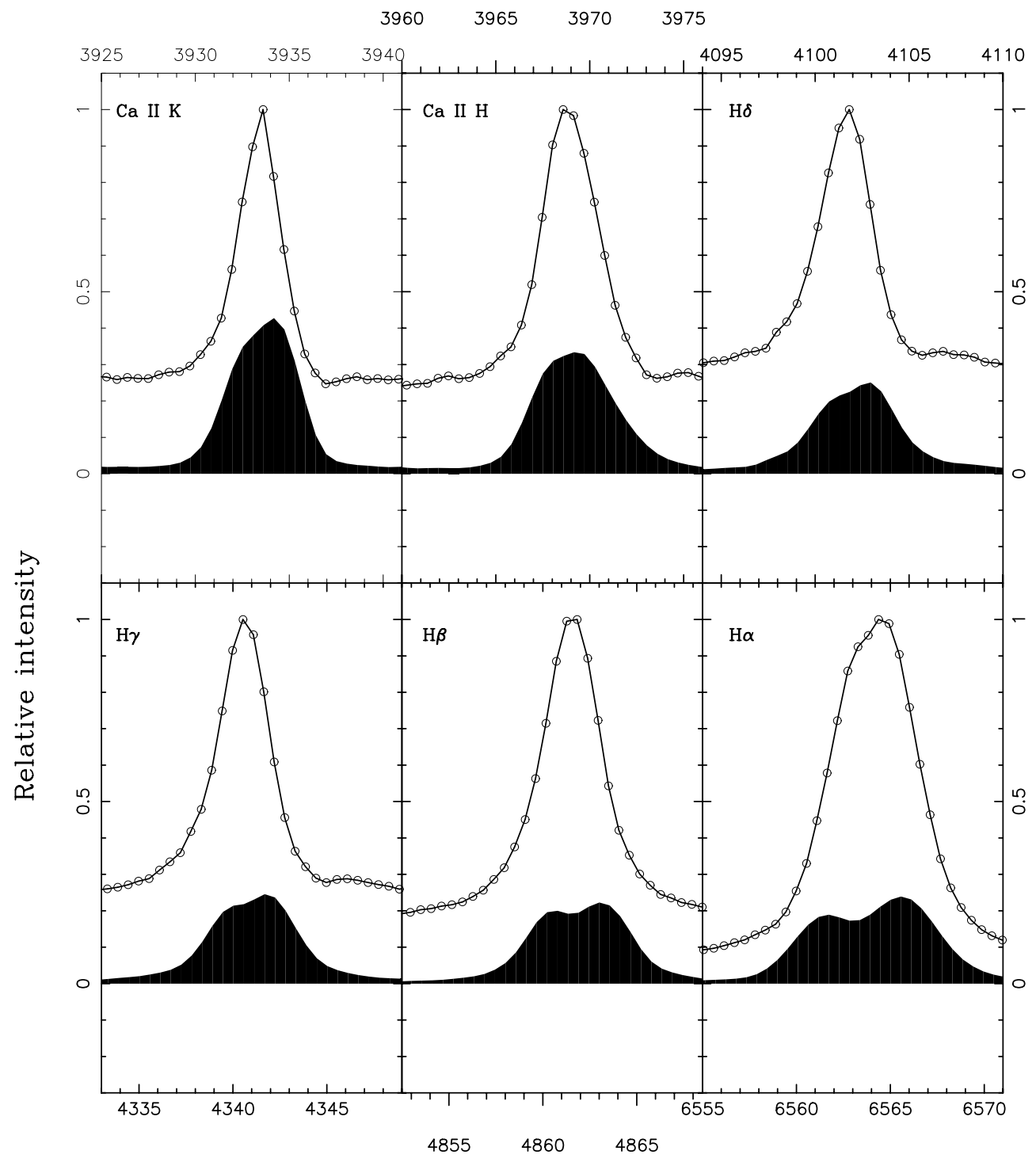

\section{Wavelength (Ang)}

Fig. 1. The standard deviation across the line profiles (shaded) compared to the average profiles (lines plus points).

seeing absorption or merely a comparative lack of emission. Hereafter we will refer to the former case as absorption and to the latter as a deficit of emission, in contrast to excess emission.

Classical T Tauri spectra typically contain a strong excess continuum which veils the stellar spectrum. This veiling continuum is variable and this will cause variations in the equivalent widths of the emission lines. Attempts were made to measure the veiling variations amongst our red spectra by measuring the variation of absorption lines (the blue spectra contained no significant absorption lines at all). These lines would be assumed to be purely photospheric. The most prominent absorption features available were the Na D lines at about $5890 \AA$ A. These revealed variations which were apparently slightly anticorrelated with the equivalent width variations of the Balmer lines. This would be expected from variable veiling, but also from filling-in of the absorption lines by extra line flux. Since the Na D lines have been seen to show some non-photospheric emission flux in other CTTS (Edwards et al. 1994), we regard our veiling estimates as unreliable and have not included them. Variations in the veiling will of course produce variations in the emission line strength across the whole profile.

The higher Balmer lines (from $\mathrm{H} \beta$ onwards) and $\mathrm{Ca}$ II $\mathrm{H}$ and $\mathrm{K}$ show clear evidence of redshifted and 
K. Smith et al.: Infall variability in the Classical T Tauri system VZ Cha

1007
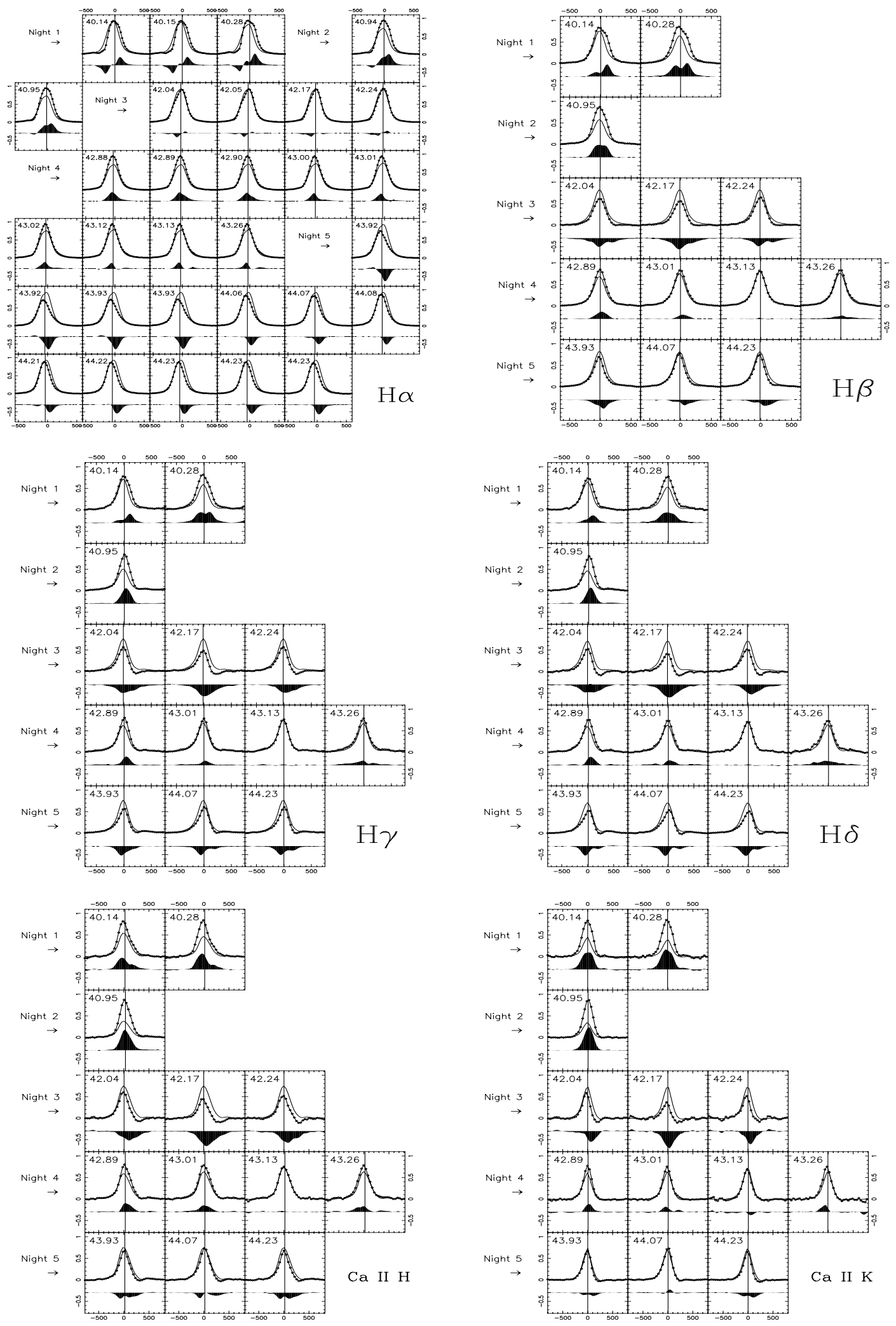

Fig. 2. The profiles of the strongest lines over the course of the observing run. The continuum-normalised spectrum is shown as a heavy solid line with points, the average profile is shown as a light solid line, and the difference between these (the residual emission) as a shaded area. The centroid of the line is also shown in each panel (vertical line). The spectra have been scaled vertically so that the maximum point of either the average or programme spectrum is set at one and the continuum level is at zero. The residuals have been displaced downwards by 0.3 for clarity. For the lines in the blue spectrum, the spectra from each night are shown on a separate line. For $\mathrm{H} \alpha$, to save space, the various nights data are shown consecutively and the separations between the successive nights of the observing run are indicated. The observation date (actually JD-2450900.0) is noted in each panel. 
blueshifted excess emission early in the run (nights one and two). $\mathrm{H} \alpha$ by contrast shows excess red emission and a deficit on the blue side of the line. A central emission component appears and grows in the $\mathrm{H} \alpha$ profile over nights one and two, whilst the blue deficit diminishes. By night three, the excess in the higher Balmer and Ca II lines has given way to a general emission deficit across the profile, with the main part in the red wing. $\mathrm{H} \alpha$ meanwhile shows small deviations from the average profile, with the red side slightly in excess and the blue side in deficit. The $\mathrm{H} \alpha$ profiles on night three appear by eye to be skewed towards the red. On night three, at $\mathrm{H} \gamma, \mathrm{H} \delta$ and the $\mathrm{Ca}$ II lines, the redshifted features dip below the continuum level and can therefore be classified as true absorption features. It should be noted here that Ca II H at $3969.6 \AA$ will be blended with $\mathrm{H} \epsilon$ at $3971.2 \AA$, so that behaviour in the red wing of $\mathrm{Ca}$ II $\mathrm{H}$ which differs from that observed at $\mathrm{Ca}$ II $\mathrm{K}$ is possibly due to variations in $\mathrm{H} \epsilon$. The profiles of these lines return to an emission excess by night four, although there is less excess emission than formerly. There is some evidence in the higher Balmer lines that the narrow central excess at the beginning of the night is replaced by a very broad excess component at $\mathrm{JD}=43.26$. On night four $\mathrm{H} \alpha$ also shows excess emission, preferentially on the blue side with a long tail into the red, and with a steady decrease in the emission near the profile centre. By the fifth night, deficits are again seen for all lines (with the possible exception of Ca II K). This time the main component is located in the blue wing for $\mathrm{H} \gamma, \mathrm{H} \delta$ and $\mathrm{Ca}$ II $\mathrm{H}$. At $\mathrm{H} \beta$ the deficit is more pronounced on the red side of the line and at $\mathrm{H} \alpha$ the deficit is entirely on the red side.

\subsection{Time series behaviour}

We computed the centroids of the lines over a wavelength range centred on the centroid of the average profile. The line centroid is

$\lambda_{\mathrm{c}}=\frac{\Sigma\left|f_{\mathrm{p}}\right| \lambda_{\mathrm{p}}}{\Sigma\left|f_{\mathrm{p}}\right|}$

where $f_{\mathrm{p}}$ are the fluxes and $\lambda_{\mathrm{p}}$ the wavelengths at each pixel. A major consideration in this calculation is the range over which the sum is taken. We chose a range of 12 pixels which ensures the entire profile is covered. The centroid of the average profile was chosen as the centre of this range as it is the most stable estimate of the true line position. Since the pixels of the average profile are interpolated to the same wavelength values as the spectrum before subtraction, the centroid of the average profile is not exactly the same for each spectrum. The variations from spectrum to spectrum due to interpolation are typically of the order of thousandths of an Ångström, and so not significant compared to the centroid variations caused by spectral variability.

An additional problem arises because the value of the average profile centroid will typically lie between pixels in the spectra. To counter this, we introduced a weighting factor to the first and last terms in each sum in Eq. (1).
These fluxes were weighted in inverse proportion to the proximity of the average spectrum centroid. For example, if the interpixel spacing is $\delta \lambda$, and the average spectrum centroid lay $0.1 \delta \lambda$ from pixel $n$ and $0.9 \delta \lambda$ from pixel $n+1$, we would assign a weight of 0.9 to the first pixel in each sum in Eq. (1) and a weight of 0.1 to the last.

The line centroid positions are marked with vertical lines in Fig. 2. We also computed the equivalent widths of the lines. Both the centroids and equivalent widths are shown in time series form in Fig. 3. Also shown in the bottom panel is the root mean square of the dispersion of the residual about the line centre. This is defined as

$W=\sqrt{\frac{\Sigma\left|f_{\mathrm{p}}\right|\left(\lambda_{\mathrm{p}}-\lambda_{\mathrm{c}}\right)^{2}}{\Sigma\left|f_{\mathrm{p}}\right|}}$,

where $\lambda_{\mathrm{p}}$ is the wavelength and $f_{\mathrm{p}}$ the residual value at each pixel and $\lambda_{c}$ is the line centroid. We will refer to this quantity as the "width" of the residual. It is insensitive to differences between absorption and emission, and also relatively insensitive to changes in the veiling, and is intended to serve as a measure of the velocity dispersion of material causing changes in the line profile. The ordinate in the lower panel of Fig. 3 has been labelled in wavelength units (on the left side) and in equivalent velocity (on the right side).

From Fig. 3 we can make a number of observations. The centroid of $\mathrm{H} \alpha$ moves from red to blue in a gradual trend over the entire observing run with no discernable shorter timescale variations. This reflects the development of deep redshifted absorption over the course of the observations (cf. Fig. 2). The other lines show more complicated behaviour. The possibility of periodic effects is discussed in a separate Sect. (4.3). There is a tendency to exhibit a blueshifted centroid on night three, indicating redshifted absorption which is also clear in Fig. 2. There is also a tendency to show redshifted centroids on night one, indicative of excess redshifted emission, and also on night five, where they seem to indicate instead a lack of blue flux.

The lower panel of Fig. 3 shows that the residual widths are generally highest on night three, when the redshifted absorption was strongest for most lines. The exceptions are $\mathrm{H} \alpha$ and $\mathrm{Ca}$ II K. The residuals show the least dispersion about the line centre on night two for all the lines except $\mathrm{H} \alpha$. The widening of the residual profiles for most lines on night four is visible in Fig. 2 and is clear for most of the lines in Fig. 3.

We have plotted the variables against one another in Fig. 4 and searched for correlations between them. Correlation coefficients and resulting significances were computed for each pair of quantities. The results appear in Table 1. It is clear that strong correlations exist between equivalent width and centroid shift for most lines. This indicates that the equivalent width variability is primarily caused by variation in the red wing of the line, since a deficit in the red will push the centroid to the blue side (a negative centroid shift in our scheme), whilst red emission 

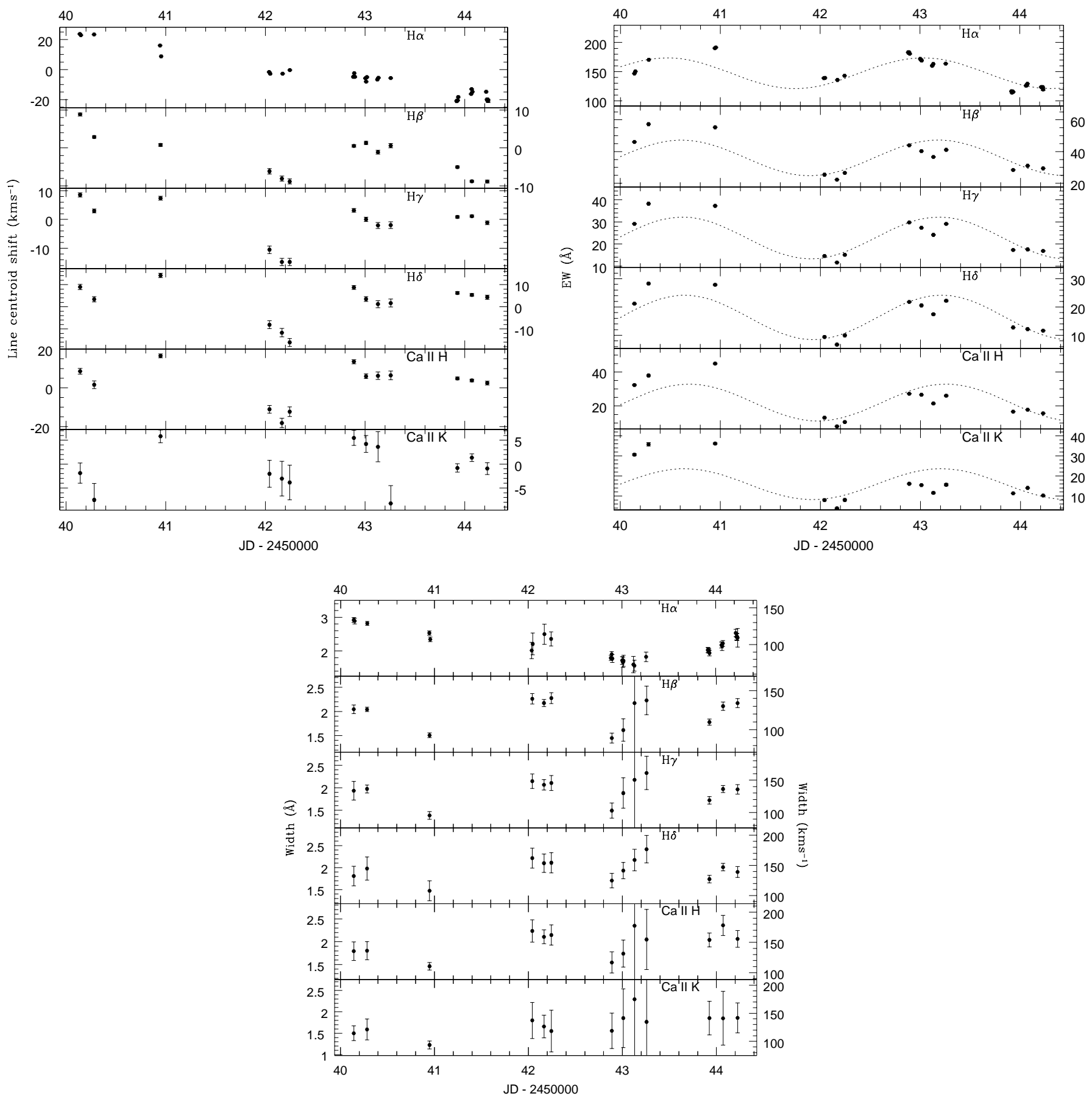

Fig. 3. The centroid shift of the spectral line (left), the equivalent widths (middle) and the residual widths (right) plotted against time. Error bars in each case are calculated from the flux errors determined in the data reduction. Positive centroid offsets indicate the line centroid was redshifted. Positive equivalent widths denote emission lines. A sine curve has been overplotted in the middle panel.

will drag the centroid to the red (a positive shift). This is consistent with the standard deviations shown in Fig. 1. The exception is Ca II K, for which the correlation is not significant.

The correlations between equivalent width and residual width and between line centroid and residual width are generally less significant. In the case of equivalent width versus residual width, a positive or negative correlation would indicate that the varying components (whether emission or absorption) occur in the line wing. Variation in both wings simultaneously would cause the largest increase in residual width. Variations in either wing, either with flux excess or flux deficit relative to the average spectrum, will cause the residual width to increase. In the equivalent width versus residual width plots, $\mathrm{H} \beta$ and Ca II H show slightly significant anticorrelations. The fact that these correlations are negative may indicate that the dominant transient features are absorption features.

Correlations between the residual width and line centroid shift probably indicate activity in the wings of the 

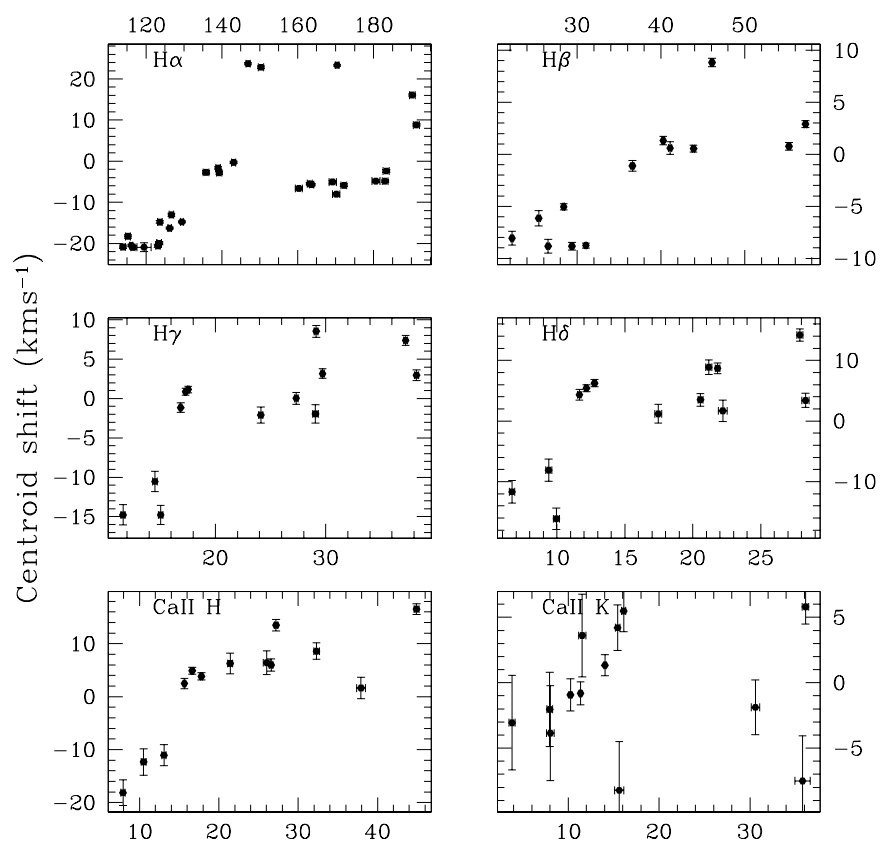

$\mathrm{EW}(\AA)$
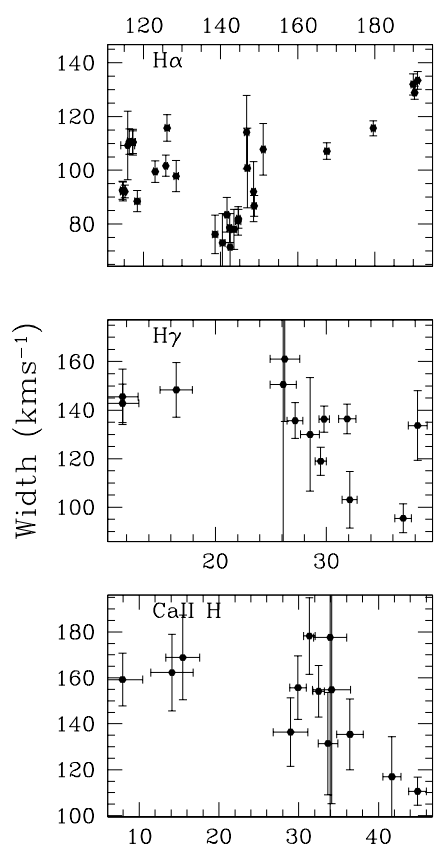

Centroid offset
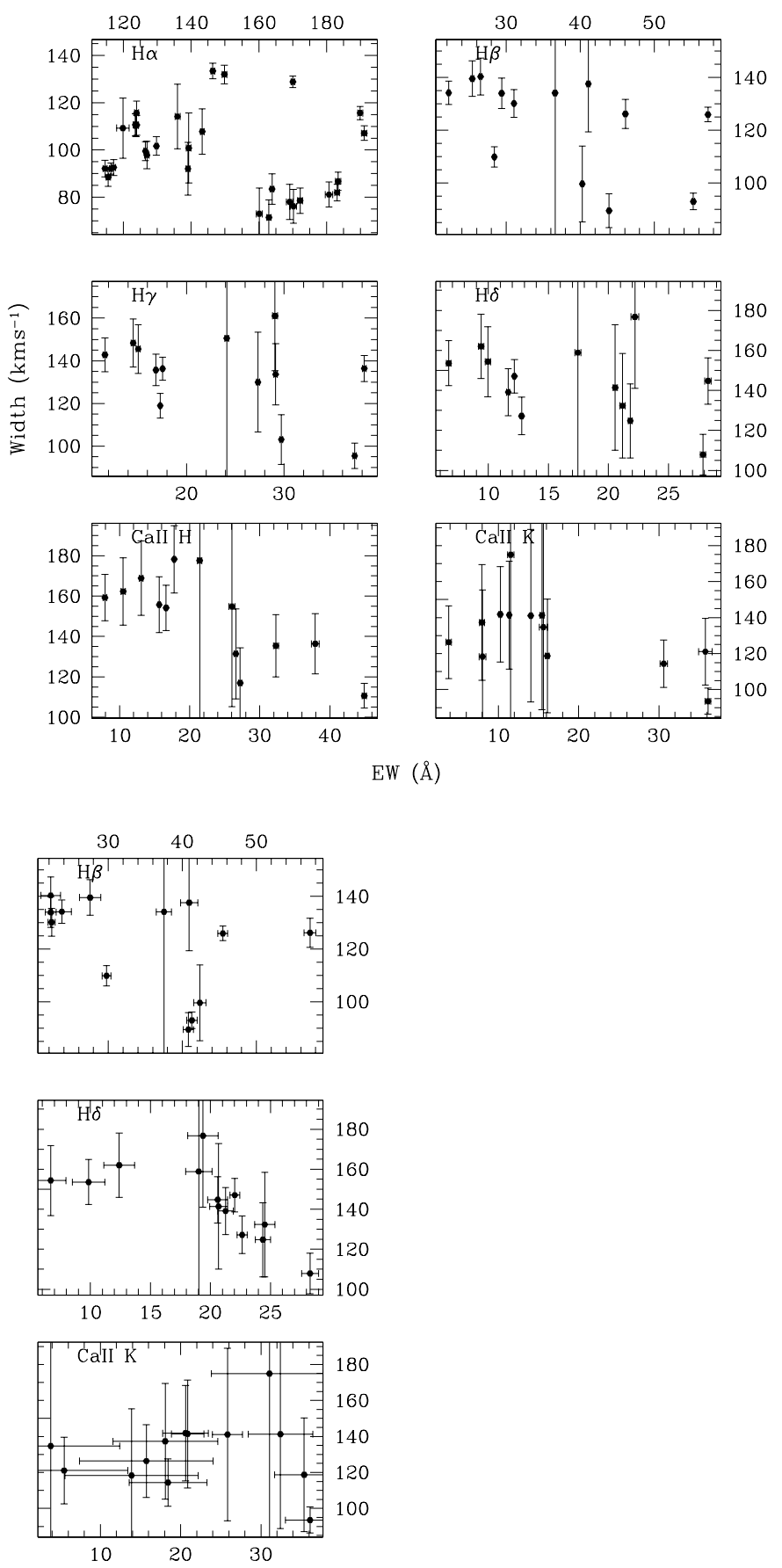

$\mathrm{EW}(\AA)$

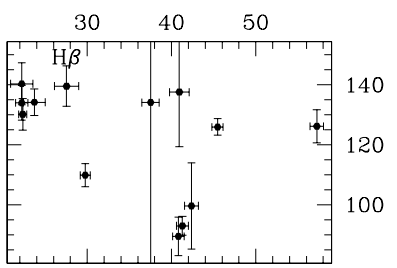

$(\AA)$

Fig. 4. Line centroid shift versus equivalent width (left panel), residual width versus equivalent width (middle), and residual width versus centroid offset (right panel).

lines. Significant anticorrelations (by the non-parametric test) are seen for $\mathrm{H} \gamma$ and $\mathrm{H} \delta$. This may indicate red flux deficits causing the line centroid to shift to the blue (negative) whilst increasing the residual width. These correlations are not immediately evident to the eye, however, and the parametric correlation coefficients are modest, perhaps indicating that the relationship is complicated by the appearance of red excess on night one or blue deficit features on night five.
To further characterise the time series behaviour of the centroids, the lines were split in two, taking the middle point to be the peak of the average spectrum at each line. The total residual on either side of the line centre was then determined. This tactic was intended primarily as a search for an anticorrelation between red shifted absorption and blue shifted emission, as reported by previous authors (Johns \& Basri 1995; Oliveira et al. 2000). Figure 5 shows correlation plots of the total red 


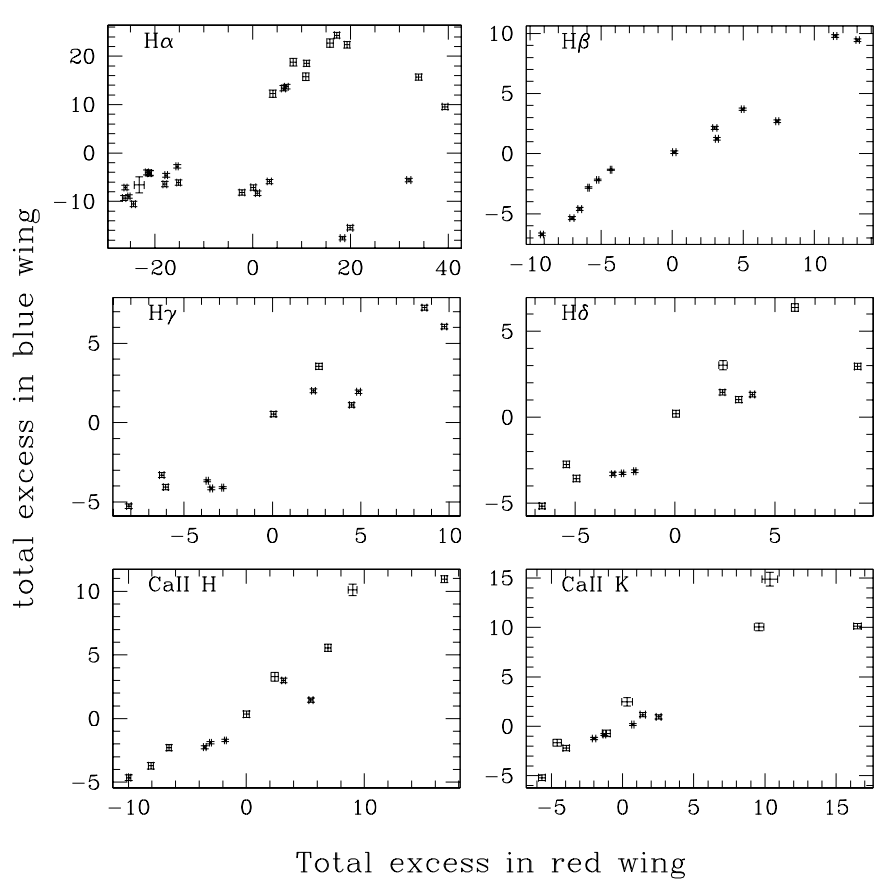

Fig. 5. The total emission in the red wing versus the total emission in the blue wing of each line. The lines are split into two halves with the centre at the position of the peak of the average profile after rebinning onto the wavelength scale of the spectrum.

residual versus the total blue residual. The red and blue sides of most of the lines vary together - as more red flux is present, so more blue flux is also observed. This situation would be expected if the line variations are driven mostly by variations in the veiling continuum. Only at $\mathrm{H} \alpha$ is this correlation not always seen. The correlation coefficients in Table 1 show that the correlations of red and blue residuals are significant at levels of $99.9 \%$ for all the lines except $\mathrm{H} \alpha$.

\subsection{Periodic effects}

Our time series' are too short and poorly sampled to make a search for periodicities in various features, but we can comment on the consistency of the data with various supposed rotational effects. As already noted, the photometric period of $\mathrm{VZ}$ Cha has been measured to be 2.56 days, and the implication is that this represents the stellar rotation period. This photometric variation could have been caused by the presence of dark photospheric spots, or by bright regions such as the accretion shocks at the magnetic poles. In the latter case we would expect to see the emission lines, which supposedly form in the accretion stream, modulated with the same period. If the photometric variation is caused by dark spots, then the line emission could be modulated with the same period or with a higher harmonic of it if there are multiple streams. In principle it is also possible that there are more spots than streams, so that the 2.56 day photometric period is a high harmonic of the true period, and the line period would then be longer.
As well as modulation of the line strengths, we might expect to see modulation of the components at various characteristic velocities as the non-axisymmetric velocity field of the accreting material rotates. This could lead to periodic behaviour in the line centroids. It should be noted that any rotational modulation would not necessarily be sinusoidal in shape.

To illustrate the discussion of periodicity for the equivalent widths, we show overplotted sine curves in the righthand panel of Fig. 3. These curves are constrained to have a 2.56 day period, as found by Batalha et al. The amplitude, phase and $y$-offset were left as free parameters. The reduced $\chi^{2}$ of these fits is very large in all cases, which would be expected from inspection of Fig. 3. It can be seen that the underlying night-to-night modulation of the equivalent widths roughly matches the expectation from the sine curve. We recomputed $\chi^{2}$ for the night-to-night variations, using nightly means of the equivalent width and Julian date and determining standard deviations of each nightly distribution separately. These are then interpreted as characterising the supposed short-timescale variations superimposed on the night-to-night variations. Night two was excluded for all the lines except $\mathrm{H} \alpha$, as there was only one point available and so it was not possible to estimate the short timescale variation.

With this procedure, the best fits are found to be $\mathrm{H} \delta$ and $\mathrm{H} \gamma$ with reduced $\chi^{2}$ of 3.4 and 4.1 respectively. $\mathrm{H} \beta$ has a reduced $\chi^{2}$ of 7 , and Ca II H and K have $\chi^{2}=14$ and 30 . $\mathrm{H} \alpha$ still has a large $\chi^{2}$ of several hundred indicating a poor fit, but for $\mathrm{H} \alpha$, the night two data is retained. Including the point from night two for the other lines, and taking the measurement uncertainty in this as $\sigma$, the reduced $\chi^{2}$ increases for all the lines, the best still being $\mathrm{H} \delta$ with a reduced $\chi^{2}$ of 8.7 . Finally we refitted $\mathrm{H} \alpha$, using the night by night data and $\sigma$ 's, and obtained a fit with reduced $\chi^{2}$ of a little over 17 . This represents the best possible sine curve fit to the $\mathrm{H} \alpha$ equivalent width time series.

Similar fits were made to the centroid time series'. These fits were in general less successful at reproducing the night to night behaviour than the fits to the equivalent width time series'. It can be seen from Fig. 3 that only $\mathrm{H} \beta$ and to a lesser extent Ca II K display the night to night variations which might be consistent with a period of around 2.5 days. Finally we note that the behaviour of the $\mathrm{H} \alpha$ centroid is entirely inconsistent with a periodic effect with a period shorter than the data set. If the $\mathrm{H} \alpha$ centroid behaviour is periodic, it must have a period of at least 10 days.

\section{Discussion}

We concluded in Sect. 4.3 that whilst the night-to-night equivalent width variations of all the lines except $\mathrm{H} \alpha$ might in part be caused by a periodic modulation with a 2.56 day period, the detailed behaviour of the equivalent width variations is inconsistent with a sine wave variation. Furthermore, the amplitude of the variation has changed between the two maxima in our dataset. This indicates 
Table 1. Correlations between various quantities. The standard parametric correlation coefficient $\left(r_{\mathrm{c}}\right)$, Spearman rank order correlation coefficient $\left(r_{\mathrm{sp}}\right)$, and the significance of the correlation $(P)$ are shown for each line. The significance level is found by a two-tailed Student's $t$ test based on the Spearman correlation coefficient. No attempt was made to test the significance of the parametric correlation.

\begin{tabular}{|c|c|c|c|c|c|c|c|c|c|c|c|c|}
\hline \multirow[b]{3}{*}{ Line } & \multicolumn{12}{|c|}{ Quantities correlated } \\
\hline & \multicolumn{3}{|c|}{$\begin{array}{c}E W \text { vs. line centroid } \\
\text { Fig. } 4 \text { top }\end{array}$} & \multicolumn{3}{|c|}{$\begin{array}{c}E W \text { vs. line width } \\
\text { Fig. } 4 \text { middle }\end{array}$} & \multicolumn{3}{|c|}{$\begin{array}{l}\text { Centroid vs. width } \\
\text { Fig. } 4 \text { bottom }\end{array}$} & \multicolumn{3}{|c|}{$\begin{array}{c}\text { Red vs. blue residuals } \\
\text { Fig. } 5\end{array}$} \\
\hline & $r_{\mathrm{sp}}$ & $P$ & $r_{\mathrm{c}}$ & $r_{\mathrm{sp}}$ & $P$ & $r_{\mathrm{c}}$ & $r_{\mathrm{sp}}$ & $P$ & $r_{\mathrm{c}}$ & $r_{\mathrm{sp}}$ & $P$ & $r_{\mathrm{c}}$ \\
\hline $\mathrm{H} \alpha$ & 0.75 & $>99.9 \%$ & 0.65 & -0.25 & $80 \%$ & -0.24 & 0.20 & $50 \%$ & 0.42 & 0.27 & $80 \%$ & 0.31 \\
\hline $\mathrm{H} \beta$ & 0.81 & $>99.9 \%$ & 0.81 & -0.63 & $98 \%$ & -0.51 & -0.53 & $90 \%$ & -0.40 & 0.99 & $>99.9 \%$ & 0.97 \\
\hline $\mathrm{H} \gamma$ & 0.81 & $>99.9 \%$ & 0.75 & -0.41 & $80 \%$ & -0.41 & -0.73 & $99.5 \%$ & -0.56 & 0.87 & $>99.9 \%$ & 0.92 \\
\hline $\mathrm{H} \delta$ & 0.56 & $95 \%$ & 0.69 & -0.38 & $80 \%$ & -0.37 & -0.85 & $>99.9 \%$ & -0.66 & 0.87 & $>99.9 \%$ & 0.85 \\
\hline Ca II H & 0.77 & $99.8 \%$ & 0.77 & -0.72 & $99 \%$ & -0.75 & -0.62 & $95 \%$ & -0.58 & 0.96 & $>99.9 \%$ & 0.92 \\
\hline Ca II K & 0.29 & $50 \%$ & 0.04 & -0.46 & $80 \%$ & -0.57 & 0.10 & $20 \%$ & 0.04 & 0.93 & $>99.9 \%$ & 0.86 \\
\hline
\end{tabular}

that there must be some other variation of the accretion system superimposed on any rotational effect.

Could we reject the idea of rotating streams altogether and rely only on variable accretion to explain the variations observed? It is of course possible to construct models based on varying accretion alone which could explain the variations, but such explanations are in our view unlikely. Many features in the profiles are seen to persist for timescales of at least one night (for example redshifted absorption on night three) or even two nights (for example the redshifted excess emission on nights one and two). Since the infall time from the inner disc edge is likely to be of order twelve hours (e.g. Paatz \& Camenzind 1996), we would expect spectral variations due to accretion of distinct blobs to occur on timescales shorter than this. The profile activity therefore suggests that accretion occurs in streams which are stable over timescales longer than the actual infall time and comparable to the rotation period. It therefore seems likely that the observed variations are indeed partly due to rotational modulation.

Two episodes of absorption in the red wings of the lines are seen. The first occurs during night three, predominantly affects $\mathrm{H} \gamma, \mathrm{H} \delta$ and $\mathrm{Ca}$ II $\mathrm{H}$ and $\mathrm{K}$, and causes genuine absorption features which dip below the continuum. A second episode begins on night four and affects $\mathrm{H} \alpha$ strongly, persisting into night five when the effect on $\mathrm{H} \alpha$ diminishes but the feature is seen at the higher Balmer lines as well.

The $\mathrm{H} \alpha$ activity is in several cases inconsistent with the activity of the other lines. A similar situation was noted by Johns-Krull \& Basri (1997) in the case of DF Tau. It seems $\mathrm{H} \alpha$ must mostly originate in a more extended region than the other lines. This is also suggested by the decoupled behaviour of the red and blue wings of $\mathrm{H} \alpha$ compared to the other lines, and is expected due to the higher optical depth of $\mathrm{H} \alpha$ compared to the other Balmer lines. Nevertheless, $\mathrm{H} \alpha$ does show a lack of flux in the red wing on night five, when the other lines undergo a weaker redshifted-absorption episode. In this instance, the behaviour seems to be more strongly coupled.

The night five observations are at a slightly earlier phase in the rotation cycle than the night three observations. It is possible therefore that we are seeing a twisted accretion stream, as suggested for SU Aur by Oliveira et al. (2000). The $\mathrm{H} \alpha$, originating mostly from the outer parts of the flow, is affected by the outer stream passing through the line of sight. This would be the effect that we observe on night five. A fraction of a rotation later, the inner part of the stream passes through the line of sight, and the optically thinner higher Balmer lines show redshifted absorption features (as on night three).

Alternatively, it is possible that there are two accretion streams causing the variations (the inclined dipole scenario). The 2.56 day period could then be one half the true rotation period of the system, and the events on night three and night five would represent the two separate streams passing through the line of sight. A nonequatorial observer's line of sight would intersect different parts of each stream, and so the variation caused by each stream would show different characterisitics. In particular, the inner part of one stream would pass through the observer's line of sight, whilst only the outer regions of the other may do so. This could lead to strong obscuration of the higher Balmer lines by rapidly moving inner material in the first stream, followed by effects seen predominantly at $\mathrm{H} \alpha$ which originates from the outer regions of the second stream. It must be remembered that such a scenario requires that an observer viewing from the northern hemisphere should have an unobstructed view of at least part of the southern hemisphere accretion stream, or vice versa. We estimate that an observer viewing from $11^{\circ}$ above the disc plane, with the disc truncated at $5 R_{*}$ and fully transparent inside that orbit, should be able to view a southern hemisphere accretion shock at an inclination of 
$10^{\circ}$ or more from the rotation axis of the star. Of course, such an observer would view the inner stream region from almost side on, and so would not see a large component of the infall velocity close to the stellar surface. Such an observer might see a large component of the infall velocity from the central portion of a curved stream, and this velocity could still be considerable (of order $100 \mathrm{~km} \mathrm{~s}^{-1}$ or more, see again Paatz \& Camenzind 1996).

In either case, we would expect the highest velocities to be associated with the inner part of the stream, and to be seen on night three. This is borne out by the lower two panels of Fig. 3, where the largest line centroid shifts are seen on night three, and also the largest residual widths. The large residual widths may indicate the phases where we see the largest dispersion of velocities in the line of sight. This is consistent with the idea that we are looking down an accretion stream during the redshifted absorption episode on night three. $\mathrm{H} \alpha$ shows some signs of behaving similarly to the other lines on night three, as the residual widths increase slightly against the general downward trend from night one to night four. Apparently, $\mathrm{H} \alpha$ is affected only slightly by an event which causes the high velocity dispersion variations in the other lines. This may be the passage through the line of sight of the inner part of an accretion stream, but with the outer part of the stream not passing directly through the line of sight. We favour this type of geometric explanation rather than an explanation based purely on optical depth, since we see from night five that $\mathrm{H} \alpha$ can be strongly affected by high velocity receding material.

\section{Summary}

Our data show that VZ Cha is an interesting object for future study. The line profiles show definite redshifted absorption features and other transient features which vary on timescales similar to the rotation timescale. We found that the variation occurred in the red wing more than in the blue wing of the line. The data we present here is not sufficiently well sampled to rigorously examine the hypothesis of periodicity for the line equivalent widths or profiles, but we can assess the viability of a rotational picture for the activity of $\mathrm{VZ}$ Cha. We find that the night-to-night variations in equivalent width are to some extent consistent with a 2.56 day periodicity, but that other variations, such as variable accretion or reconnection, are necessary to account fully for the behaviour. It is unlikely that rotational effects are entirely unimportant as the observed lifetimes of varying profile components are longer than or comparable to the infall time from the inner disc, and also comprise a significant fraction of the rotation period. We found that the red and blue variations, probably representing accretion and outflow respectively, were correlated for all lines except at $\mathrm{H} \alpha$, where there were episodes of anticorrelated behaviour. This suggests that $\mathrm{H} \alpha$ originates in a more extended region than the higher Balmer lines and samples parts of the accretion and outflow streams which are affected differently by the system's rotation.

Acknowledgements. We thank the staff at SSO and ANU for providing the facilities on which this paper is based. We thank also the referee, Christopher Johns-Krull, for his helpful comments which have allowed us to considerably improve this paper.

\section{References}

Appenzeller, I., Jankovics, I, \& Krautter, J. 1983, A\&AS, 53, 291

Basri, G., Marcy, G. W., \& Valenti, J. A. 1992, ApJ, 390, 622

Batalha, C., Quast, G. R., Torres, C. A. O., et al. 1998, A\&AS, 128,561

Bonnell, I. A., Smith, K. W., Meyer, M., et al. 1998, MNRAS, 299, 1013

Drissen, L., Bastien, P., \& St-Louis, N. 1989, AJ, 97, 814

Edwards, S., Hartigan, P., Ghandour, L., \& Andrulis, C. 1994, AJ, 108, 1056

Ghez, A. M., McCarthy, D. W., Patience, J. L., \& Beck, T. L. 1996, ApJ, 481, 378

Ghosh, P., \& Lamb, F. K. 1978, ApJ, 223, 83

Guenther, E. W., Lehmann, H., Emerson, J. P., \& Staude, J. 1999, A\&A, 341, 768

Gullbring, E., Hartmann, L., Briceno, C., \& Calvet, N. 1998, ApJ, 492, 323

Hartmann, L., Hewett, R., \& Calvet, N. 1994, ApJ, 426, 669

Herbig, G., \& Bell, K. R. 1988, Lick Obs. Bull., 1111

Hessmann, F. V., \& Guenther, E. W. 1997, A\&A, 321, 497

Johns, C. M., \& Basri, G. S. 1995, ApJ, 449, 341

Johns-Krull, C., \& Basri, G. S. 1997, ApJ, 474, 433

Johns-Krull, C., Valenti, J. A., Hatzes, A. P., \& Kannan, A. 1999, ApJ, 501, L41

Kappelman, N., \& Mauder, H. 1981, ESO Messenger, 23, 18

Kenyon, S., Hartmann, L., Hewett, R., et al. 1994, AJ, 107, 2153

Königl, A. 1991, ApJ, 370, L39

Krautter, J., Appenzeller, I., \& Jankovics, I. 1990, A\&A, 236, 416

Mauder, H., \& Sosna, F. M. 1975, Inf. Bull. Var. Stars, 1049

Miller, K. A., \& Stone, J. M. 1997, ApJ ,489, 890

Muzerolle, J., Calvet, N., \& Hartmann, L. 1998, ApJ, 492, 743

Oliveira, J. M., Foing, B. H., van Loon, J. Th., \& Unruh, Y. C. 2000, A\&A, 362, 615

Paatz, G., \& Camenzind, M. 1996, A\&A, 308, 77

Shu, F. H., Najita, J., Ostriker, E., et al. 1994, ApJ, 429, 781

Smith, K. W., Bonnell, I. A., \& Lewis, G. F. 1995, MNRAS, 276,263

Smith, K. W., Lewis, G. F., Bonnell, I. A., \& Bunclark, P. S. 1998, MNRAS, 289, 151

Smith, K. W., Lewis, G. F., Bonnell, I. A., Bunclark, P. S., \& Emerson, J. P. 1999, MNRAS, 304, 367

Ultchin, Y., Regev, O., \& Bertout, C. 1997, ApJ, 486, 397 